\title{
Is It Time to Revisit Pediatric Postdischarge Home Visits for Readmissions Reduction?
}

\author{
Andrew S Kern-Goldberger, MD*, S Ryan Greysen, MD, MHS, MA²,3
}

'Section of Pediatric Hospital Medicine, Children's Hospital of Philadelphia, Philadelphia, Pennsylvania; ${ }^{2}$ Section of Hospital Medicine, Division of General Internal Medicine, Perelman School of Medicine, University of Pennsylvania, Philadelphia, Pennsylvania; ${ }^{3}$ Leonard Davis Institute of Health Economics, The Wharton School, University of Pennsylvania, Philadelphia, Pennsylvania.

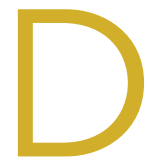

espite concerted national efforts to decrease pediatric readmissions, recent data suggest that preventable and all-cause readmission rates of hospitalized children remain unchanged. 'Because some readmissions may be caused by inadequate postdischarge follow-up, nurse (RN) home visits offer the prospect of addressing unresolved clinical issues after discharge and ameliorating patient and family concerns that may otherwise prompt re-presentation for acute care. Yet a recent trial of this approach, the Hospital to Home Outcomes (H2O) trial, ${ }^{2}$ found the opposite to be true: participants receiving home nurse visits had higher reutilization rates than did participants in the control group. This raises interesting questions: Is it time to revisit postdischarge outreach as an intervention to reduce pediatric readmissions-and even pediatric readmissions altogether as an outcome metric?

In this issue of the Journal of Hospital Medicine, Riddle et al ${ }^{3}$ explored the perspectives of key stakeholders to understand the factors driving increased reutilization after postdischarge home visits in the $\mathrm{H} 2 \mathrm{O}$ trial and obtained feedback for improving potential interventions. The investigators used a qualitative approach that consisted of telephone interviews with 33 parents who were enrolled in the $\mathrm{H} 2 \mathrm{O}$ trial and in-person focus groups with 10 home care RNs involved in the trial, 12 hospital medicine physicians, and 7 primary care physicians (PCPs). Inductive thematic analysis was used to analyze responses to open-ended questions through a rigorous, iterative and multidisciplinary process. Key themes elicited from stakeholders included questions about the clinical appropriateness of reutilization episodes; the influence of insufficiently contextualized "red flag," or warning sign, instructions given to parents in facilitating reutilization; the potential for hospital-employed home care nurses to inadvertently promote emergency department rather than PCP follow-up; and escalation of care exceeding that expected in a PCP office. Stakeholders suggested the intervention could be improved by enhancing postdischarge communication between home care RNs, hospital medicine physicians, and PCPs; tailoring home visits to specific clinical, patient, and family scenarios; and more clearly framing "red flags."

\footnotetext{
*Corresponding Author: Andrew S Kern-Goldberger, MD; Email: kerngoldba@ email.chop.edu; Telephone: 267-303-2638; Twitter @ASKGoldberger.
}

Received: May 5, 2020; Accepted: May 6, 2020

๑) 2020 Society of Hospital Medicine DOI 10.12788/jhm.3460
We welcome the work of Riddle and colleagues in exposing the elements of home visits that may have led to increased utilization, and their proposed next steps to improve the intervention-enhancing contact with PCP offices and focusing interventions on specific populations-unquestionably have merit. We agree that this may be particularly true in children with medical complexity (a population that was excluded from this study), who have unique discharge needs and account for over half of pediatric readmissions. ${ }^{4}$ However, we suggest that the instinct to refine the design of the study intervention should be weighed against alternative possibilities: that postdischarge interventions are simply not effective in decreasing reutilization or, at the very least, that the findings of the $\mathrm{H} 2 \mathrm{O}$ trial should not lead us to invest the resources required to further discern the efficacy of postdischarge interventions.

This counter-intuitive possibility is only compounded by the fact that reutilization rates were not improved in the study group's H2O II trial, a follow-up study that focused on postdischarge nurse telephone calls as the intervention of interest ${ }^{5}$; and indeed, the results of these two, well-designed negative trials have been previously cited to propose postdischarge nurse contact as a potential target of deimplementation efforts. ${ }^{6}$ In the pediatric population, in which caregivers rather than patients themselves are generally responsible for seeking out care, postdischarge outreach may inevitably escalate concerning findings that will result in reutilization. Instead, perhaps the $\mathrm{H} 2 \mathrm{O}$ study findings should prompt a broader exploration for alternative solutions to pediatric readmission reduction. One such solution could build on the finding by Riddle et al that stakeholders perceive ambiguity in whether discharging physicians, or rather PCPs, have ownership of clinical issues after discharge. Rather than asking visiting RNs to triangulate between inpatient and outpatient physicians, developing systems to directly integrate PCPs in the hospital discharge process for select patients-for instance, through leveraging the rapid expansion of telemedicine services during the COVID-19 crisis-may promote shared understanding of a patient's illness trajectory and follow-up needs.

Importantly, the authors also noted that despite the findings of increased reutilization, parents who received home visits expressed their wishes to receive home visits in the future. While not a central finding of the study, this validates a hypothesis expressed in prior work by the $\mathrm{H} 2 \mathrm{O}$ study group: "Hospital quality readmission metrics may not be well aligned with family desires for improved postdischarge transitions." Given that efforts to reduce pediatric readmission have been 
largely unsuccessful and that readmission events are relatively uncommon in the general pediatric population, ${ }^{4}$ the parental wishes resonate with existing calls in the literature to consider looking beyond readmissions reduction in isolation as a quality metric. In contrast to the increasing presence of hospital reimbursement penalties among state Medicaid agencies for readmissions, a shift in focus toward outcome measures that are patient- and family-centered is imperative. ${ }^{1,7}$ If home visits are not ultimately a solution to pediatric reutilization reduction, they may nonetheless still enable families to effectively manage the concerns that families endorse following discharge, including medication safety and social hardships. ${ }^{8}$

In summary, Riddle et al not only provided important context for the unexpected outcome of a well-designed randomized clinical trial but also provided a rich source of qualitative data that furthers our understanding of a child's discharge home from the hospital through the perspective of multiple stakeholders. While the authors offer well-reasoned next steps in narrowing the intervention population of interest and enhancing connections of families with PCP care, it may be time to broadly revisit postdischarge interventions and outcomes to identify new approaches and redefine quality measures for hospital-to-home transitions of children and their families.
Disclosures: The authors report no conflicts of interest.

\section{References}

1. Auger KA, Harris JM, Gay JC, et al. Progress (?) toward reducing pediatric readmissions. J Hosp Med. 2019;14(10):618-621. https://doi.org/10.12788 /jhm.3210

2. Auger KA, Simmons JM, Tubbs-Cooley HL, et al. Postdischarge nurse home visits and reuse: the hospital to home outcomes $(\mathrm{H} 2 \mathrm{O})$ trial. Pediatrics. 2018;142(1):e20173919. https://doi.org/10.1542/peds.2019-0092

3. Riddle SW, Sherman SN, Moore MJ, et al. A qualitative study of increased pediatric reutilization after a postdischarge home nurse visit. J Hosp Med. 2020;15:518-525. https://doi.org/10.12788/jhm.3370

4. Berry JG, Toomey SL, Zaslavsky AM, et al. Pediatric readmission prevalence and variability across hospitals. JAMA. 2013;309(4):372-380. https://doi. org/10.1001/jama.2012.188351

5. Auger KA, Shah SS, Tubbs-Cooley HL, et al. Effects of a 1-time nurse-led telephone call after pediatric discharge: the $\mathrm{H} 2 \mathrm{O} \| \mathrm{I}$ randomized clinical trial. JAMA Pediatr. 2018;172(9):e181482. https://doi.org/10.1001/jamapediatrics.2018.1482

6. Bonafide $C P$, Keren R. Negative studies and the science of deimplementation. JAMA Pediatr. 2018;172(9):807-809. https://doi.org/ 10.1001/jamapediatrics.2018.2077

7. Leyenaar JK, Lagu T, Lindenauer PK. Are pediatric readmission reduction efforts falling flat? J Hosp Med. 2019;14(10):644-645. https://doi. org/10.12788/jhm.3269

8. Tubbs-Cooley HL, Riddle SW, Gold JM, et al. Paediatric clinical and social concerns identified by home visit nurses in the immediate postdischarge period. J Adv Nurs. 2020;76(6):1394-1403. https://doi.org/10.1111/jan.14341 